\title{
Congrès SOFFCOMM 2012
}

\author{
D. Nocca \\ C) Springer-Verlag France 2012
}

\section{Chers collègues}

C'est avec un grand plaisir que l'équipe du CHRU de Montpellier vous accueille à Montpellier pour le congrès annuel de la Société Française et Francophone de Chirurgie de l'Obésité et des Maladies Métaboliques.

Pour la première année, la SOFFCOMM a décidé d'inviter tous les professionnels de santé impliqués dans la prise en charge de l'obésité sévère ou morbide à participer à ces journées d'échanges et de formation consacrées à un des fléaux sanitaires actuels de notre société. En effet, vous n'êtes pas sans savoir que l'obésité morbide touche plus de 600.000 personnes en France et que les conséquences socio-économiques inhérentes aux comorbidités liées à l'obésité (diabète type 2, HTA, syndrome d'apnées du sommeil, problèmes articulaires, cancer (eh oui!)... sont dramatiques. La chirurgie est elle le traitement idéal de cette vraie maladie chronique qu'est l'obésité? Surement pas! Cependant, c'est à l'heure actuelle, le seul traitement efficace pour traiter les patients atteints d'obésité sévère.

Qu'on soit pour ou contre, des milliers de patients sont opérés dans les services de chirurgie bariatrique chaque année et notre devoir est d'assurer une qualité de soins irréprochable. Pour ce faire une prise en charge pluridisciplinaire est incontournable. Chaque professionnel de santé (endocrinologue, médecin nutritionniste, diététiciennes, psychologues, psychiatres, infirmières, kinésithérapeutes...) à un rôle thérapeutique important et doit être impliqué dans l'équipe bariatrique. Le rôle du médecin généraliste est encore obscur. Etant le praticien qui connaît le mieux le patient à traiter, il devrait être au centre de la réflexion sur l'indication opératoire. Cependant, pour des raisons d'information souvent erronée sur la chirurgie bariatrique (ce n'est pas de la chirurgie esthétique!!!) ou d'emploi du temps sur-

\footnotetext{
D. Nocca $(\bowtie)$

Président du congrès SOFFCOMM 2012

e-mail : david.nocca@sfr.fr
}

Directeur du comité scientifique SOFFCOMM

Coordinateur de l'équipe de chirurgie bariatrique

du CHRU Montpellier chargé leur implication est trop faible à l'heure actuelle dans nos réseaux de soins. A nous de travailler sur des propositions (télémédecine, formation médicale continue...) pour leur faire une place privilégiée.

Concernant la SOFFCOMM, je voulais souligner l'évolution extraordinaire de cette jeune société savante qui est en plein essor. Plus de 140 abstracts ont été reçus pour l'organisation du congrès 2012, ce qui prouve un vif intérêt de nos collègues chirurgiens mais aussi médecins. De plus notre société a, grâce à l'investissement de ses dirigeants (merci Pr Mouiel et Pr Chevallier notamment!) contribué à l'organisation nationale de cette nouvelle discipline médico-chirurgicale. Les nombreuses réunions à la HAS, à la CNAM, au ministère ont permis de valider des recommandations de bonnes pratiques qui doivent (si elles sont respectées!) éviter les abus du passé. Temps de préparation trop court, IMC trop bas et absence de concertation pluridisciplinaire doivent être bannies de nos pratiques pour que notre discipline soit crédible!

De plus, le plan national pour lutter contre l'obésité, édité récemment, devrait permettre d'aider à mieux gérer la filière de soins, ainsi qu'à favoriser la recherche et la prévention dans ce domaine. Il paraît évident que ce plan obésité ne peut se concevoir que par, encore une fois, une collaboration pluridisciplinaire dans laquelle tous les professionnels de santé travailleront de concert.

Enfin, je voudrais finir par un mot pour remercier l'équipe pluridisciplinaire de chirurgie bariatrique du CHRU Montpellier, récemment élue "centre spécialisé pour la prise en charge de l'obésité sévère" par l'ARS Languedoc Roussillon.

Merci donc à Patrick, Charles, Jean Michel, Eric, Medhi, Marius, Sylvie, Frédérique, Virginie, Laurence, Nathalie, Valérie, Florence, Brigitte (oui même toi), Moez, Sophie, Samir, Carole, Marie Christine, Elizabeth ... et à notre doyen le Pr Jacques Bringer (un des fondateurs de la chirurgie bariatrique au CHU Montpellier) qui a compris, il y a plus de 15 ans, l'intérêt d'une collaboration médico-chirurgicale dans ce domaine.

Bon congrès! 